\title{
Detection of rolling bearing degradation using infrared thermography
}

\author{
by A. MAZIOUD*, L. IBOS*, A. KHLAIFI*, J.F. DURASTANTI* \\ *Laboratoire CERTES (EA 3481) IUT of Sénart, Lieusaint, 77127, France
}

\section{Abstract}

Our study deals with the quantification of the energy dissipated by a defect of spalling on the outside ring of a rolling bearing. A previous work showed a correlation between the thermal heating and the vibratory level generated by the progressive appearance of the defect.

First, we propose a numerical model of the heat transfers In a second time, we developed an experimental setup. The results obtained in this first step are encouraging. Indeed, the defect generated in the rolling bearing leads to a quantifiable heating of the surface, and the numerical model and the experiments allow quantifying the involved flux.

\section{Introduction}

The activity of maintenance of the industrial installations has been used since twenty years and the diagnosis techniques are more and more sophisticated. Several techniques of defect detection are at the disposal of the maintenance engineers today. Among these, we can note the infrared thermography for the detection of defects of electrical or mechanical origin; the analysis of lubricants; the ultrasound detection; the mechanical vibrations.

The last technique uses signal analysis tools which have seen their field of application widening year after year. We can find in [1] the necessary elements to implement the classic signal analysis tools, such as: Fourier Transform, Hilbert transform (for the search for amplitude modulations andlor frequency), as well as the determination of scalar indicators such as: effective value, factor of crest and Kurtosis.

Submitted to radial effort and / or axial which can vary in direction during time, a rolling bearings (figure 1) gets tired under the influence of a variation of load on its rolling elements.

At the end of 19th century, Hertz [2] established the relations which allow to calculate the constraints and the deformations which undergo two surfaces which run without sliding the one on the other one.

These cutting constraints which undergo the rolling elements entail, at the end of a certain number of hours of functioning, a loss of metal in surface called spalling. This spalling (figure 2) can occur a priori on one of three rolling surfaces (rolling element, track of the outside ring, the track of the internal ring).

This defect of spalling is then going to degenerate to provoke the other sources of spalling and entail the progressive destruction of the rolling bearings.

During the passage of the rolling element on the spalling zone, a shock is going to occur. This impact is repeated in a periodic way. The frequency of repetition of the impact depends on the geometry of the rolling bearing and on the speeds of rotation.

\section{Vibratory analyses}

Many methods are used in signal analysis tools to prevent defect in the rolling bearing.

\subsection{Speed vibration}

The vibration is measured in radial direction by an accelerometer piezoelectric. From this measure, we calculate the speed vibration (by integration of the signal), then the effective value of this speed in the frequency interval of $1-10 \mathrm{kHz}$. We obtain the global level in $\mathrm{mm} / \mathrm{s}$.

These measures are compared with the limit values appearing in the international recommendations as the standard ISO 10816 used by SKF Company. By putting back results of the measurement according to time, we can appreciate the evolution of the vibrations and estimate the date of replacement of the rolling bearing. diagnostic.

This method allow as to have first indication of the rolling bearing degradation but we can't make a real

\subsection{The crest factor}

We can be earlier warned of the rolling bearing defect by using the same material as previously, but by measuring the crest factor on frequency interval of $1-10 \mathrm{kHz}$ :

$$
C F=\frac{A_{\text {crest }}}{A_{\text {eff }}}
$$

CF : crest factor

$A_{\text {crest }}$ : Maximal amplitude of the signal

$A_{\text {eff }}$ : Effective amplitude of the signal 
At the beginning this report is appreciably constant. When a localized defect develops, the small resultant shocks increase level of crest, but have a weak influence on the effective value. The level of crest increases then until a maximum. When the defect of the rolling bearing increases, there are more impacts generated in every passage of rollings. The effective level thus increases, whereas the level of crest remains unchanged. In the end life of the rolling bearing, the factor of crest can fall again to its initial value because the effective value has Increased in his turn.

The major defect of this indicator is to present approximately the same values in both cases Extremes (new and end state of life of the rolling bearing). Only its evolution is thus significant.

\subsection{The kurtosis}

The kurtosis, allows do detect the presence of shocks in the measured signal. More the value of the kurtosis is raised, more the presence of shocks is important. For indication, an absence of shocks gives a kurtosis of 1.5 while this value can rise to a several units in a rolling bearing with Spalling echo defect. The kurtosis must be calculated after filtering the signal in a range containing a frequency.

The kurtosis is given by the following relation:

$$
K=\int_{-x}^{x} \frac{(x-\bar{x})}{\sigma^{4}} P(x) d x
$$

$\mathrm{x}$ : instantaneous amplitude of the signal

$x:$ mean value of $x$

$\mathrm{P}(\mathrm{x})$ : Probability of appearance of $\mathrm{x}$

$\sigma:$ Standard deviation

\subsection{Spectrum analysis}

We transformed by Fourrier the measured temporary signal to a frequencies representation, called spectrum. The shocks on the spectrum are manifested by peaks regularly spaced out by the frequency of the defect (fbe, fbi or fer). These peaks will have an amplitude more raised to the neighborhood of the machine frequencies echo. For a first measure, it is advised to choose a wide band $(0-20 \mathrm{kHz})$ to identify well the rang of echo.

\subsection{Envelope Detection}

When these periodic peaks are not clearly visible on the spectrum, we proceed to a filtering of the signal around a frequency of echo. After demodulating of the filtered signal, we study finally the spectrum. The periodic peaks are visible then more clearly.

The manufacturers of the rolling bearing supply the values of the bearing frequencies. It is just enough to indicate the reference of the concerned rolling bearing and the frequencies of rotation of both rings.

Table 1. Frequency of spalling defekt

\begin{tabular}{|l|l|l|l|l|}
\hline $\mathrm{N}_{\mathrm{i}}$ rpm & $\left.\mathrm{N}_{\mathrm{e}} \mathrm{rpm}\right)$ & $\mathrm{f}_{\mathrm{be}}(\mathrm{Hz}$ & $\mathrm{f}_{\mathrm{bi}}(\mathrm{Hz})$ & $\mathrm{f}_{\mathrm{er}}(\mathrm{Hz})$ \\
\hline 1490.4 & 0 & 76.84 & 121.88 & 109.96 \\
\hline
\end{tabular}

$\mathrm{N}_{\mathrm{i}}$ : Speed rotation of the internal ring;

$\mathrm{N}_{\mathrm{e}}$ : Speed rotation of the outside ring;

$f_{b e}$ : Impact frequency if spalling is on the outside ring;

$\mathrm{f}_{\mathrm{bi}}$ : impact frequency if spalling is on the internal ring;

$f_{e r}$ : Impact frequency if spalling is on rolling element,

Remark: these repeated shoks are going to incite the machine and to make it vibrate with particular frequencies (frequency of echo).

The frequencies are given by the following formulae:

$$
f_{b e}(H z)=\frac{n}{2} f_{r}\left(1-\frac{B D}{P D} \cos \beta\right)
$$

Frequency of spalling Defect on the outside ring :

$$
f_{b i}(H z)=\frac{n}{2} f_{r}\left(1+\frac{B D}{P D} \cos \beta\right)
$$


Frequency of spalling Defect is on rolling element:

$$
f_{e r}(H z)=\frac{P D}{B D} f_{r}\left[1-\left(\frac{B D}{P D} \cos \beta\right)^{2}\right]
$$

Frequency of spalling Defect on the casing:

$$
f_{b e}(H z)=\frac{n}{2} f_{r}\left(1+\frac{B D}{P D} \cos \beta\right)
$$

Where :

$\mathrm{n}$ : number of rolling elements

$\mathrm{fr}$ : speed of rotation of the shaft

$\mathrm{BD}$ : diameter of the rolling elements in $\mathrm{mm}$

PD: diameter primitive of the bearing in $\mathrm{mm}$

$\beta$ : angle of contact in radians

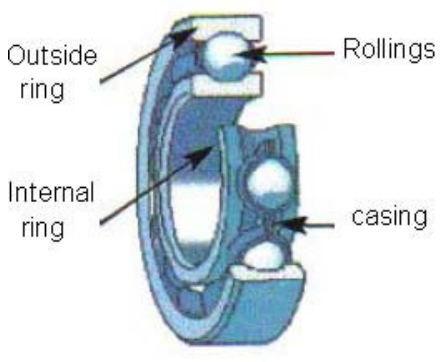

Fig 1. Rolling bearing

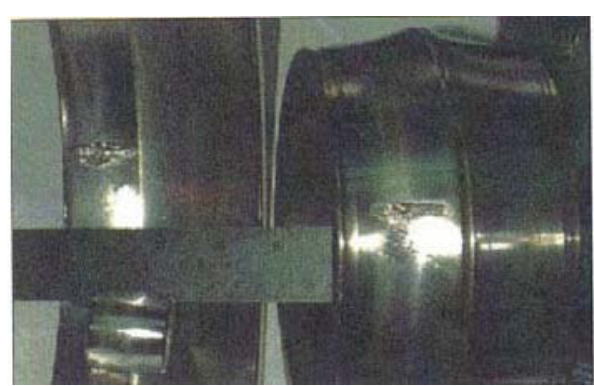

Fig 2. Spalling defect

\section{Infrared Thermography}

The Infraed thermography is the technique which allows, from the radiation emited by a scene, of adapted equipments and techniques of mastery of the measure situation, to obtain the spatial and temporal distribution of the temperatures of this observed scene.

- It's very performent Tool to diagnose any type of heating origin as :

- Electric maintenance

- Buildings

- Furnaces and boilers

- Mechanics and friction

- A fluid Problem

In our study, the measure of temperature based on infrared thermography allows us to detect the presence of abnormally warm zones on the surface of the bearing.

We propose here to establish a link between the temperature rise and the rise of the vibratory level of a mechanical component in the course of degradation. Our study particularly concerns the detection of the appearance of a defect of spalling on a rolling bearing [3]

\subsection{Equation of the thermography}

It is necessary to remind that an infrared camera does not measure directly a temperature of surface. The detectors of cameras are sensitive to the luminance emitted by the studied surface. This luminance is naturally dependent on the temperature of surface of it, But other parameter of influence enter stake.

The equation (1), corresponding to the fundamental equation of the infrared thermal imaging connect the luminance measured in the temperature of the surface studied $\mathrm{T}_{0}$.

$$
L=T \varepsilon L^{\circ}\left(T_{0}\right)+T \rho L^{\circ}\left(T_{\text {env }}\right\}+(I-T) L^{\circ}\left(T_{a}\right)
$$

The measured luminance $L$ contains three terms: a term of emission, a term of reflection consider as a parasite and a term due to a presence of a not perfectly transparent atmosphere between the aimed surface and the camera. The notation $L^{\circ}(T)$ corresponds to the luminance of a body with temperature $T$ considered as a black body, according to Stefan's law:

$$
\begin{aligned}
& L^{\circ}(T)=\sigma T^{4} \\
& \text { Were, } \sigma=5,67 \times 10^{-8} \text { W.m. } \mathrm{m}^{-2} \mathrm{~K}^{-4}(2)
\end{aligned}
$$


In equation (3) should be written by using spectral greatnesses, in a given wavelength $\lambda$. To simplify the estimations of temperature, it is more interesting to use total greatnesses, that is on all the band strip wavelength in which the camera is sensitive. This simplification is based on numerous hypotheses, among which the fact that observed bodies (solid surfaces and atmosphere) are "grey" in the spectral band of the camera, their radiative properties are independent from the wavelength.

The appropriate emission, issue of the studied surface depends on its temperature of surface, but also on the emissivity $\varepsilon$ of this one. It thus seems clearly here that a bad knowledge of the emissivity of the aimed surface can generate an important error on the temperature of surface. The term of reflection "causes parasites" depends on the average temperature of the radiative environment $T_{\text {env }}$ and on the factor of reflexion $\rho(\rho=1-\varepsilon$ for an opaque surface) of the aimed surface.

The term of the atmosphere emission depends on the temperature of the ambient air $T_{\mathrm{a}}$. and the factor of transmission $\mathrm{T}$ of the atmosphere in the spectrum of wavelength of the camera. The transmittance of the atmosphere also intervenes in the first two terms (appropriate emission and parasite effect) of the equation (1), because an toning down of the luminance emitted or reflected by the wall is observed if the placed atmosphere enters the aimed scene and The camera is not perfectly transparent.

From measuring the luminance, $\mathrm{L}$, and after mastering the situation of measurement (Knowledge of all the enumerate parameters) and the calibration of the thermal camera, we shall arrive to measure correctly the temperature by infrared thermography.

\section{The methodology}

The methodology is described below (see also figure 3):

Periodic impacts are generated by a spalling zone located between one of both rings of the rolling bearing (an impact in every passage of a rolling on the spalling zone).

These periodic impacts are going to excite the mechanical system structure (movement, connection with the machine frame ...).

The whole bearing cap is thus going to vibrate at its echo frequency.

The damping of the vibration has the effect to transform a part of the damping vibratory energy into heat.

This heat creation induces a rise in the ring temperature, more particularly on its external surface.

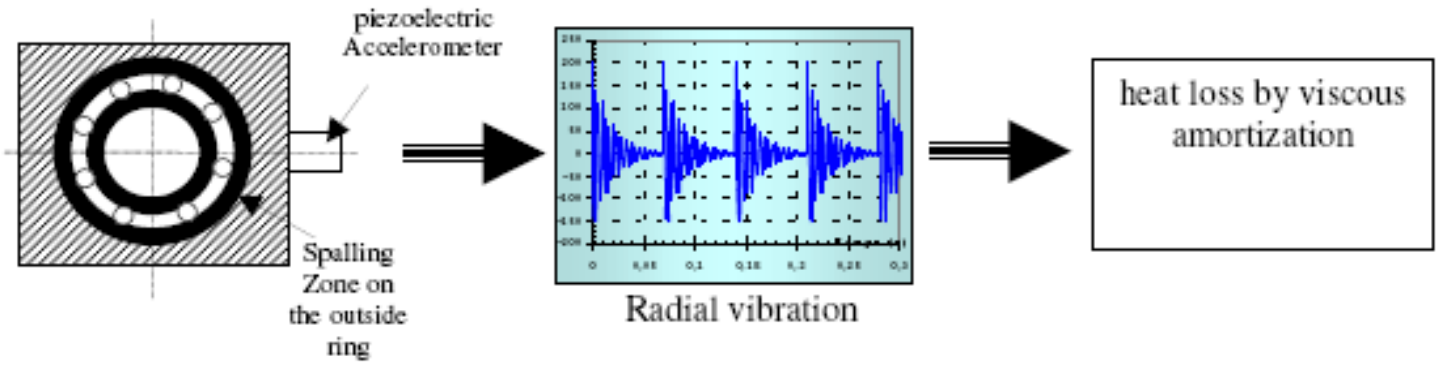

Fig 3. Rise of temperature of the rolling bearing due to the presence of a spalling zone on the outside ring

To summarize, we think that the measurement of this temperature rise might allow us to estimate the vibratory energy quantity generated by the periodic impacts, and therefore to estimate the level of the rolling degradation.

\section{The test platform}

In this part of the study, we put in place a setup which allows us to study the thermal behaviour of rolling bearing SKF 6002. The objective is to measure the thermal contact resistance between the rings and the rollings for different temperatures, speeds and loads.

The Setup is equipped with a three-phase electric engine controlled by an automaton, consisting of a rotating axis, two bearing and a measuring cell made of aluminium in the which is found the rolling bearing type SKF 6007. The internal ring of the rolling bearing to be studied is assembled on system of heating.

This system is formed by a hollow cylinder in Teflon on which comes to rest a heating thermal resistance $(R=20 \Omega)$ fed by a tension generator through a system of coal collectors. A hollow cylinder made of aluminium was adjusted on the thermal resistance to standardize the surface temperature.

The system of heating as well as the internal ring of the rolling bearing are placed on a rotating axis.

Nine drillings of diameter $0,6 \mathrm{~mm}$ and of $7 \mathrm{~mm}$ depth were made by electro-erosion on the rings, eight equally spaced drillings on the outside rings and one on the internal ring.

The setup so realized is equipped with a flux metre placed between the system of heating and the internal ring to quantify the injected heat flux. Nine thermocouples type $\mathrm{K} \emptyset 0,5 \mathrm{~mm} \times 150 \mathrm{~mm}$ measure the temperatures in the drillings.

Three transmitters of signals 2 wires Ref $\left(747-154\right.$, scale of measure between $\left.-25,150^{\circ} \mathrm{C}\right)$ fixed on a disk placed on the rotating axis allow us to receive the signals delivered respectively by the flux-meter and the 
thermocouple placed on the rotating ring, These amplified signals will be sent afterwards to the acquisition system of coals collectors.

\section{Simulation of defects}

We can simulate an outside ring defect of the rolling bearing by more or less tightening a screw with radial effort (figure 5)

A sensor which measures strength (type Entran model ELHS-B1-5KN, scale of measure $5000 \mathrm{~N}$ ) placed between the outside ring of the rolling bearing and the screw allows us to quantify the force exerted.

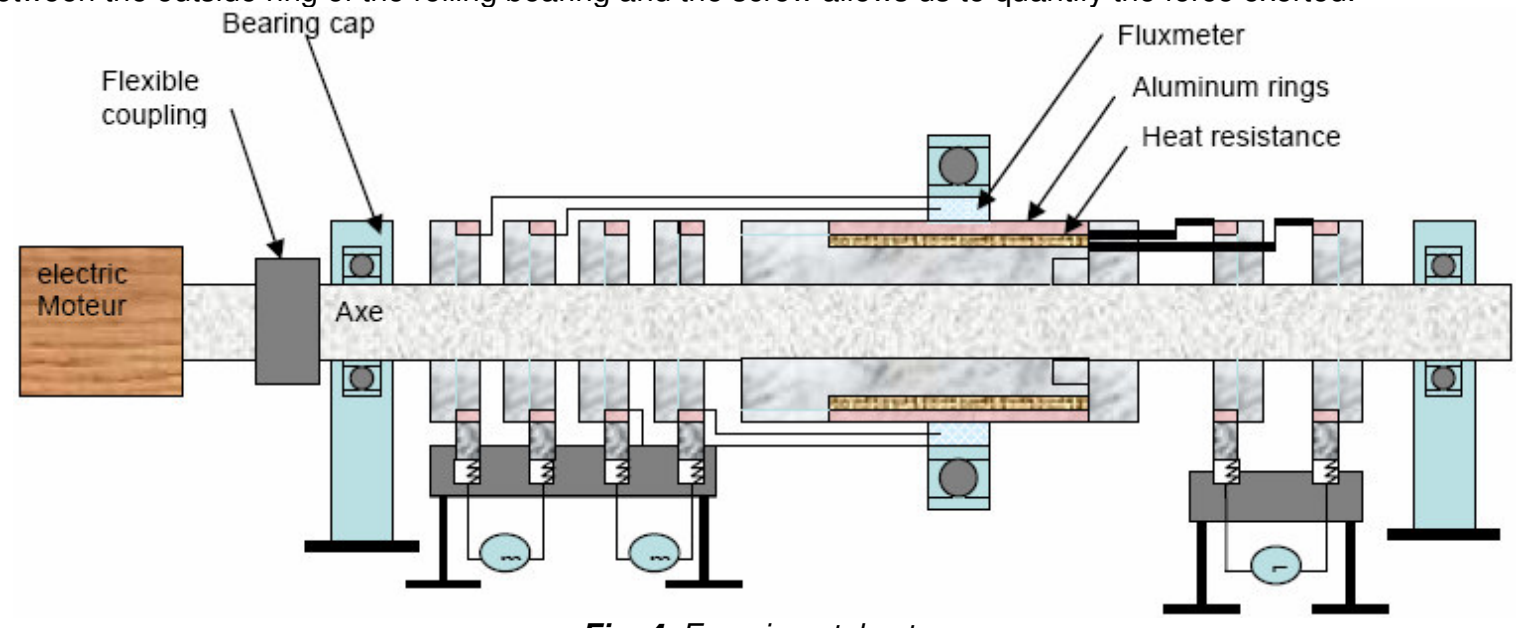

Fig. 4. Experimental setup

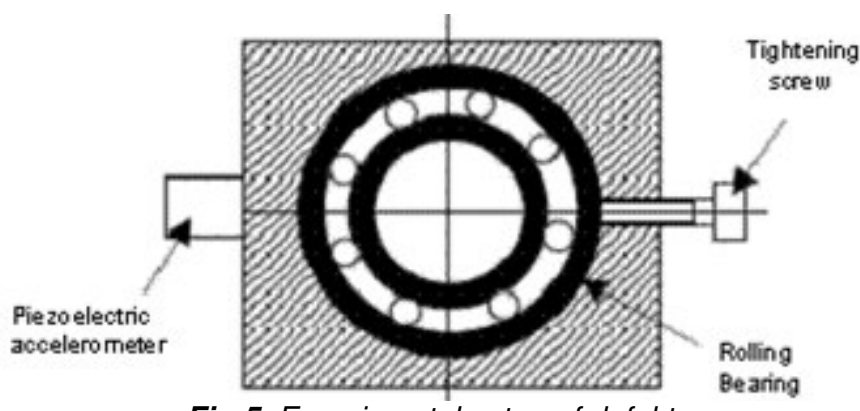

Fig 5. Experimental setup of defekt

An accelerometer measures the vibration in the radial direction. An infrared thermal camera is used to measure the bearing cap exterior surface temperature (figure 6). (We painted a part of the surface of the bearing cap using a high emissivity painting for minimizing the effect of the reflection and reduce the errors of measure.
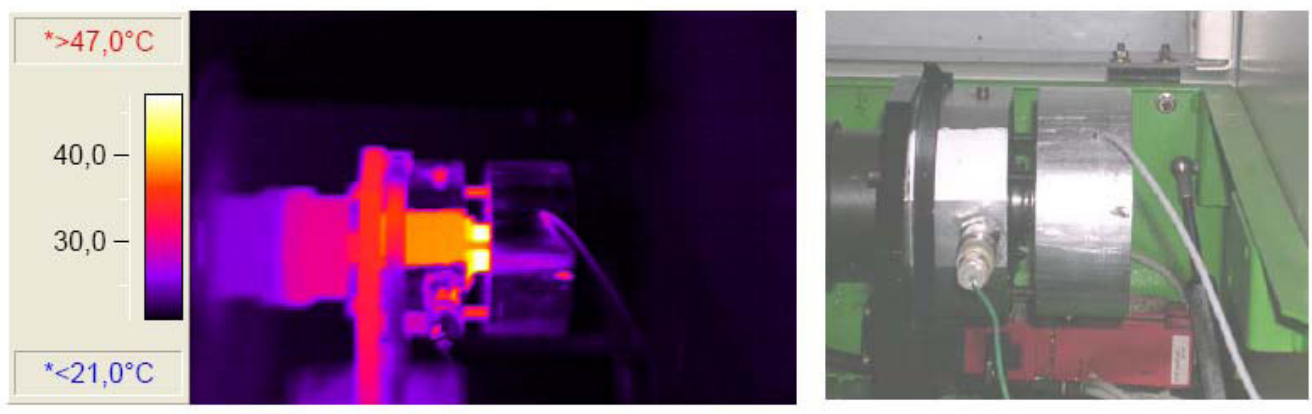

Fig 6: The bearing cap seen by infrared camera

The rotation speed of the axis is maintained constant: $1500 \mathrm{rpm}$

We have measured, for various forces applied by the tightening screw:

The vibratory level (effective value in $\mathrm{mm} / \mathrm{s}$ ),

The temperature of the external surface of the bearing cap.

The obtained results are given in figure 7. 


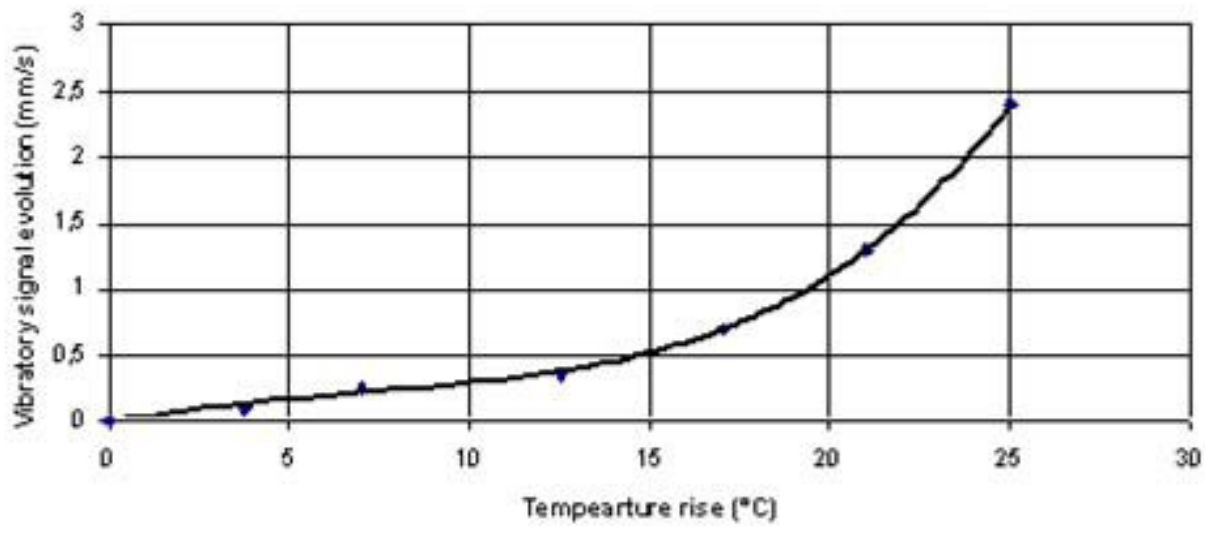

Fig 7. Evolution of the temperature of the bearing cap according to the vibratory level

There is a clear correlation between mechanical and thermal effects.

We have also established a thermic modelling of the bearing cap of the rolling bearing.

\section{Numeric model}

We are interested in determining the temperature field in the rolling bearing, the bearing cap and the rotation axis. For this purpose, we used a software based on the finite volume method [Fluent].

We remember that the presence of a provoked defect leads to a characteristic vibratory level transformed into abnormal heating of the system, and thus the generation of a heat fluxes adding to the nominal heat production. We wish to verify whether this phenomenon induces a rise of the surface temperature that can be detected by infrared thermography [4]. The modeling part thus consists in changing the fluxes generated and to measure the spatial distribution of the temperature.

The modeling hypotheses are the following:

We assimilate the rollings to a ring;

We suppose that the contacts between rings and rollings are perfect;

The rotation speeds of the internal ring and the rollings are identical.

Heat production is uniform in the ring

We present in figure 8 the chosen mesh; it is a three-dimensional structured mesh using parallelepiped cells realized under GAMBIT so as to optimize the number of cells with the aim of improving the precision and reducing the computing time, the number of cells is 16079 .
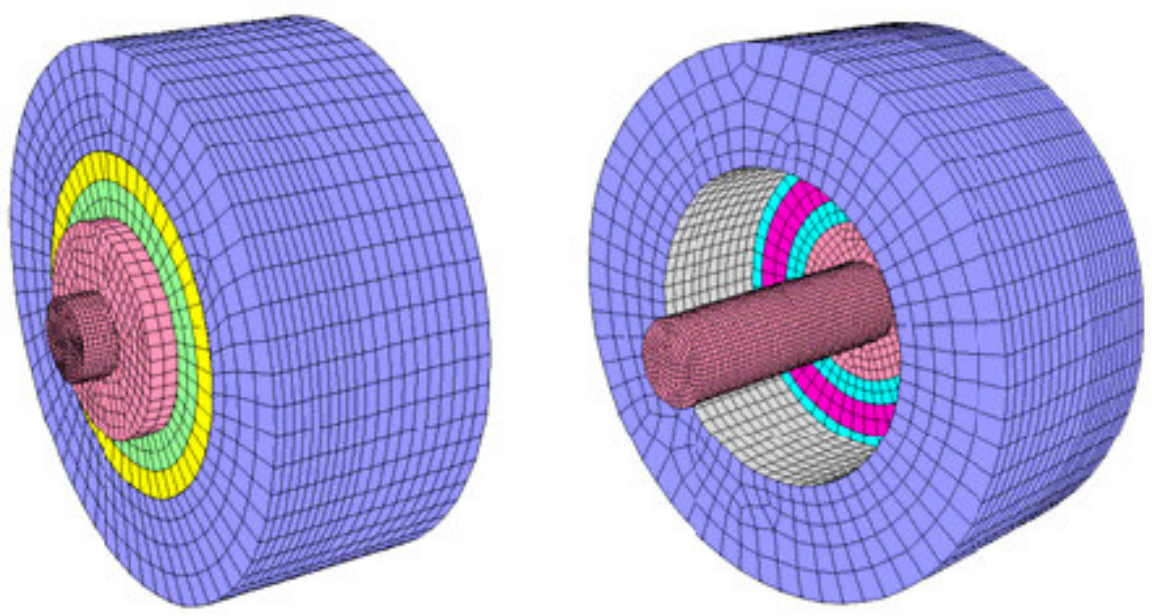

Fig 8. Meshing of the experimental device

The computing conditions are as follows:

Ambient temperature: $20^{\circ} \mathrm{C}$

Internal and external side convection exchange coefficients: $10 \mathrm{~W} / \mathrm{m}^{2} / \mathrm{K}$

Convection exchange coefficients on the lower and upper faces: $10 \mathrm{~W} / \mathrm{m}^{2} / \mathrm{K}$

Speed of rotation of the axis: $1500 \mathrm{rpm}$

The bearing cap is made of aluminum with thermal conductivity: $\lambda=202.4 \mathrm{~W} / \mathrm{m}$. K 
The rolling bearing and the axis are made of steel with thermal conductivity: $\lambda=16.27 \mathrm{~W} / \mathrm{m}$. K

The figure 9 represents the temperature field on a section of the experimental device for various values of the power generated inside the rolling bearing.

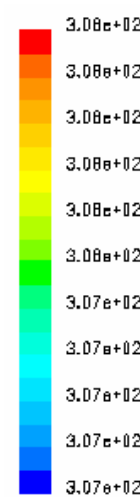

$$
\mathrm{P}=14.8 \mathrm{~W}
$$

$3.35 \mathrm{e}+02$

$3.358+02$

$3.34 \mathrm{e}+02$

$3.348+102$

$3.33 \mathrm{e}+02$

$3.32 \mathrm{~B}+12$

$3.32 \mathrm{e}+02$

$3.319+02$

$3.310+02$

$3.30 \mathrm{E}+02$

3.300+12

$$
\mathrm{P}=66.6 \mathrm{~W}
$$
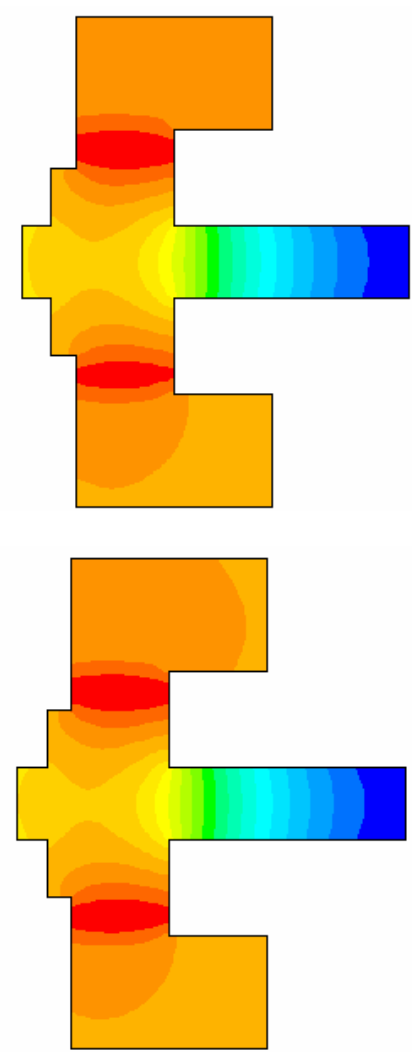
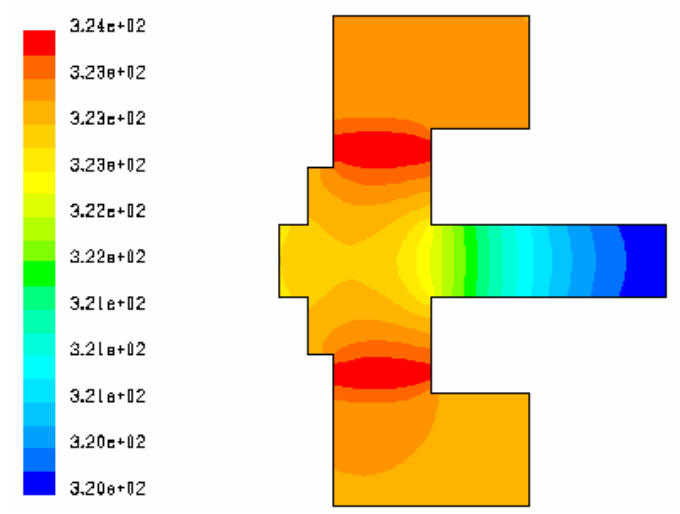

$$
\mathrm{P}=44.4 \mathrm{~W}
$$
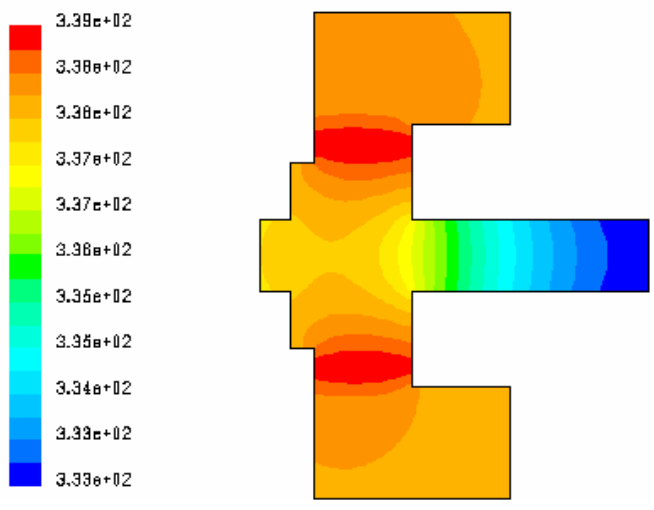

$\mathrm{P}=74 \mathrm{~W}$

Fig 9. Field of temperatures computed within the bearing cap with movement

In figure 10, we present the evolution of the difference $\Delta T$ between bearing cap and ambient temperatures according to the power generated inside the rolling bearing.

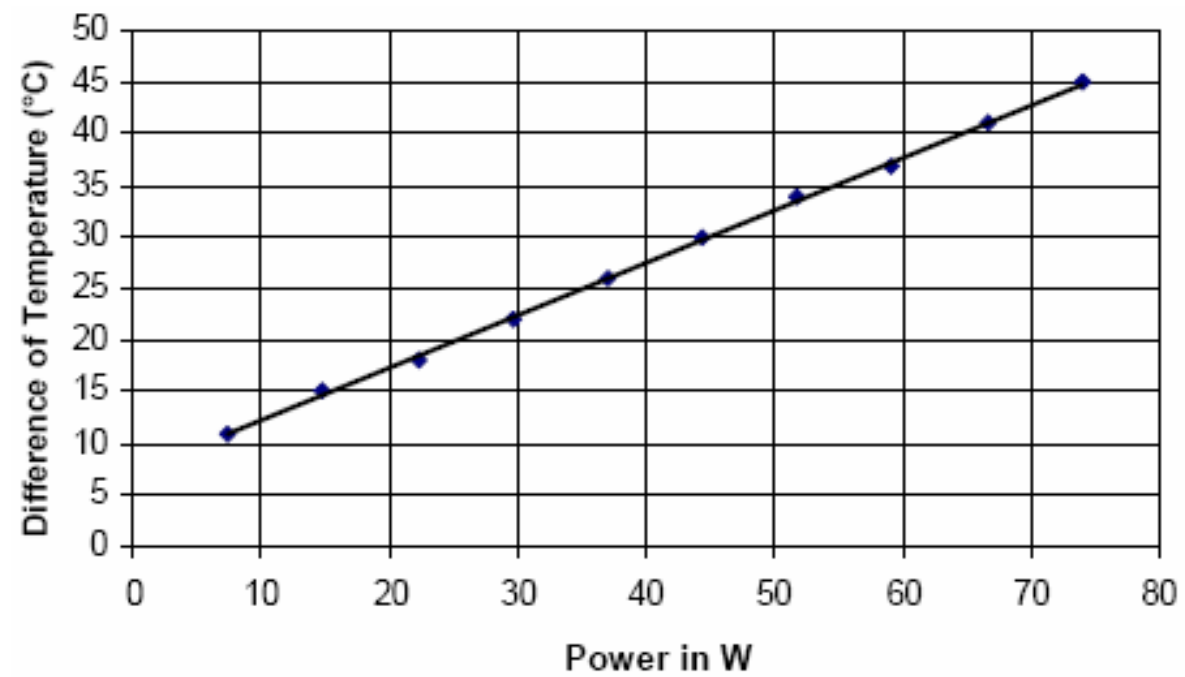

Fig 10. Calculated evolution of $\Delta T$ as a function of the power dissipated by the rolling bearing

As we could expect, the evolution of $\Delta \mathrm{T}$ is proportional to the power dissipated by the heat source. The slope coefficient is equal to $0.51^{\circ} \mathrm{C} / \mathrm{W}$. 


\section{Conclusion}

The first results obtained are encouraging. Indeed, on the one hand the vibratory defect engendered within the rolling bearing leads to a quantifiable heating of the surface; on the other hand, numeric model highlights a correlation between vibratory defect and heat production but also allows to quantify the involved fluxes.

This work will have to continue following to two axes. A mechanical model should complete concerning the analysis of the mechanical phenomenon, the approach, purely experimental up to now,, in order to predict heat generation from the defect characteristics.

Also, the thermal model must be improved by taking into account other parameters like for instance the contact resistances between different mobile parts of the rolling bearing.

\section{REFERENCES}

[1] A. Boulenger, C. Pachaud, Analyse vibratoire en maintenance préventive, DUNOD, 2003.

[2] Hertz H., On the Contact of Rigid Elastic Solids and on Hardness, Miscellaneous Papers, Mac-Millan, London, 1896.

[3] Barret, M., Durastanti, J. F., Madani, K., Diagnostic vibratoire basé sur la transformée en ondelettes, Congrès CNRIUT'03, Mai 2003, Tarbes (France).

[4] Padjani, D., mesure par thermographie infrarouge, ADD, 1989. 